\title{
Preliminary efficacy of fluticasone delivered by a novel device in recalcitrant chronic rhinosinusitis *
}

\author{
F.S. Hansen ${ }^{1}$, P.G. Djupesland ${ }^{2}$, W.J. Fokkens ${ }^{1}$ \\ 1 Academic Medical Centre, Amsterdam, The Netherlands \\ 2 OptiNose AS, Oslo, Norway
}

\begin{abstract}
SUMMARY Objective: To assess whether delivery of fluticasone propionate using a novel bi-directional delivery device (Opt-FP) offers therapeutic benefits in patients with chronic rhinosinusitis (CRS).

Methods: A prospective, single centre, randomized, double-blind, placebo (PBO)-controlled, parallel group study was conducted in adult subjects $(n=20)$ with CRS without nasal polyps or only cobblestoned mucosa. Subjects received Opt-FP $400 \mu \mathrm{g}$ or placebo twice daily for 12 weeks ( $n=10 /$ group). Outcome measures included symptom scores, RSOM-31, CRS VAS, nasendoscopy, peak nasal inspiratory flow (PNIF) and magnetic resonance imaging (MRI).

Results: Endoscopy score for oedema showed a highly significant and progressive improvement (12 weeks (median scores): Opt-FP -4.0, PBO -1.0, $p=0.015)$. PNIF increased significantly during Opt-FP treatment compared to placebo (4 weeks: $p=0.006 ; 8$ weeks: $p=0.03$ ). After 12 weeks MRI scores in the Opt-FP group improved against baseline $(p=0.039)$ and a non-significant trend was seen versus placebo. The nasal RSOM-31 subscale was significantly improved with Opt-FP treatment (4 weeks: $p=0.009,8$ weeks: $p=0.016,12$ weeks: NS). Sense of smell, nasal discomfort and combined score were all significantly improved $(p<0.05)$. The Opt-FP was well tolerated.
\end{abstract}

Conclusions: The OptiNose breath-actuated bi-directional delivery device administering fluticasone propionate (400 $\mu \mathrm{g}$ b.i.d.) is an effective and well tolerated treatment for recalcitrant CRS.

Key words: fluticasone, bi-directional delivery, chronic rhinosinusitis, paranasal sinus disease

\section{INTRODUCTION}

Chronic rhinosinusitis (CRS) is a persistent $(>12$ weeks with no resolution of symptoms) inflammation of the nose and paranasal sinuses characterized by two or more of the following symptoms: blockage/congestion, discharge with anterior/post nasal drip, facial pain/pressure, a reduction or loss of smell, along with either endoscopic signs: polyps, mucopurulent discharge from the middle meatus, oedema/mucosal obstruction primarily in the middle meatus and/or CT changes, mucosal changes within the ostiomeatal complex and/or sinuses ${ }^{(1)}$. It is a common condition and the impact on quality of life can be considerable ${ }^{(2,3)}$.

A number of different intranasal corticosteroids have been found to be effective in reducing polyp size and associated symptoms in moderate and severe polyposis, but only two contradictory studies exist in CRS patients with no or minimal polyposis $^{(4)}$.

Conventional nasal sprays, drops and nasal powder inhalers are suboptimal for efficient delivery to the middle meatus ${ }^{(5,6)}$. Bi-directional delivery using the OptiNose device offers an alternative method with highly superior delivery to target areas beyond the nasal valve, including the middle meatus ${ }^{(7)}$. Delivery to the middle meatus is considered essential to achieve clinical effects in CRS with a topical steroid ${ }^{(8)}$. The Opt-FP, which contains a multi-dose spray pump, is primed and positioned in one nostril with the mouthpiece in the mouth. The user blows through the device which causes the soft palate to close, separating the nasal and oral cavities, and triggering the spray pump. The airflow generated in the nose expands the narrow nasal passages and the communication located behind the nasal septum during soft palate closure before exiting through the other nostril in the opposite direction (bi-directional flow).

The aim of this study was to investigate the efficacy and safety of Opt-FP in subjects with CRS without nasal polyps or only cobblestoned mucosa.

\section{MATERIALS AND METHODS}

Study design

This prospective, randomized, double-blind, placebo-controlled, parallel group study enrolled adult subjects with recalcitrant CRS at a single ENT clinic in the Netherlands 
Table 1. Inclusion and exclusion criteria.

\section{Inclusion Criteria}

1. Male and female subjects aged between 18 and 65 years of age.

2. Subjects with chronic rhinosinusitis defined as at least 12 weeks history of two or more of: blockage/congestion, discharge: anterior/post nasal drip, facial pain/pressure, reduction or loss of smell and either mucopurulent discharge from the middle meatus or oedema/mucosal obstruction primarily in the middle meatus.

3. Women of child-bearing age must be neither pregnant nor lactating and must use a medically acceptable form of contraception.

4. No clinically significant abnormal serum biochemistry, haematology and urine examination values on screening.

5. In the clinicians judgement fit and able to participate in the study.

6. Subjects to provide written informed consent to participate in the study.

7. Subjects must have correctly completed the daily Diary during the run-in period.

8. Willing and able to comply with the study requirements.

9. Subjects must have verified airflow through both nostrils and an ability to close their soft palate.

10. The subject must have the ability to trigger the breath- actuation mechanism of an OptiNose device in accordance with the Instructions For Use.

\section{Exclusion Criteria}

1. Visible nasal polyps on endoscopy, except cobblestoned mucosa.

2. Surgical treatment for nasal polyps during the previous 3 months.

3. A diagnosis of cystic fibrosis.

4. Other disease likely to interfere with the study parameters, evidence of any serious or unstable concurrent disease or psychological disorder.

5. A hypersensitivity or contraindication to steroids.

6. Depot or oral steroids during the previous 2 months.

7. A requirement for more than $1000 \mu \mathrm{g}$ beclomethasone (or equivalent) per day for the treatment of asthma.

8. Subjects taking inhaled steroids whom have not been on a stable dose for 3 months or more.

9. Unable to cease treatment with intranasal steroids, or intranasal sodium cromoglycate, decongestants or antihistamines at the Screening Visit.

10. Currently receiving leukotriene receptor antagonists, nasal atropine or ipratropium bromide or neuroleptics.

11. Subjects using devices that dilate the nostrils to improve nasal breathing.

12. Subjects being treated with ritonavir or other potent CYP3A inhibitors.

13. A history of operations where metal objects were used and retained in the body or those with a pacemaker or other implants and artificial heart valves.

14. A history of a penetrating injury to the eye with a metal object or who have worked with metal at high speed.

15. A history of drug or alcohol abuse.

16. An inability to communicate well with the Investigator.

17. Subjects with a cleft palate.

18. Subjects who have participated in a New Chemical Entity study within the previous 16 weeks or marketed drug study within the previous 12 weeks.
(Academic Medical Centre (AMC), Amsterdam). All subjects gave written informed consent to participate in the study, which was conducted in accordance with the Declaration of Helsinki and the principles of Good Clinical Practice. The study was reviewed and approved by the medical ethics committee of the AMC.

Male and female outpatients aged 18-65 years who had a diagnosis of CRS were screened. The subjects had either no nasal polyps or only cobblestoned mucosa and thus fulfilled the EP3OS criteria of CRS ${ }^{(2)}$.

Inclusion and exclusion criteria for the study are presented in Table 1. Subjects using saline rinses were permitted to continue to do so. Loratadine $10 \mathrm{mg}$ tablets were provided as rescue medication for the relief of troublesome symptoms. If a subject experienced a severe acute nasal blockage the investigator could authorize the use of a short course of oxymetazoline drops or spray for a maximum of 7 consecutive days and a maximum total of 10 days during the treatment period. Oxymetazoline was not to be used within 24 hours of a scheduled study visit.

Following a 14-16 day treatment-free run-in, subjects who met the eligibility criteria were randomized 1:1 to receive Opt-FP
$400 \mu \mathrm{g}$ or placebo b.i.d. for 12 weeks. Subjects attended the clinic at the beginning and end of the run-in period and after 4 , 8 and 12 weeks of treatment. A follow-up visit was made 2 weeks after the end of treatment.

The Opt-FP and placebo devices were identical in appearance. The spray pump in the Opt-FP contained an aqueous suspension of fluticasone propionate (FP) $0.1 \% \mathrm{w} / \mathrm{w}$ in an aqueous medium containing microcrystalline cellulose and carboxymethylcellulose sodium, benzalkonium chloride, EDTA disodium salt dehydrate, dextrose anhydrous and polysorbate 80. Placebo matched FP exactly, except for the active ingredient. The devices delivered $100 \mu \mathrm{L}$ aqueous suspension per actuation. To deliver a dose of FP $400 \mu \mathrm{g}$ b.i.d. or placebo, subjects made two administrations per nostril, morning and evening.

All subjects were trained in the use of the device. Nasal patency and ability to close the soft palate were confirmed at screening. Compliance was assessed at each visit by examining the devices for use and reviewing treatment administrations recorded in the diary cards. 


\section{Efficacy assessments}

The following assessments were undertaken at the clinic visits pre-dose and after 4, 8 and 12 weeks of treatment:

1. Rhinosinusitis Outcome Measure 31 (RSOM-31). Subjects completed this fully validated quality of life scale containing 31 items in 7 domains. The nasal subgroup includes 7 questions. A reduction in the average total symptom impact score $>1$ is considered clinically relevant ${ }^{(9)}$.

2. Subjective symptom scores including nasal blockage, nasal discomfort and rhinitis symptoms were recorded by subjects in a diary each morning and evening from screening through to the end of treatment using the following scoring system: 0 (none), 1 (mild - symptoms present but not troublesome), 2 (moderate - symptoms frequently troublesome but not interfering with daily activity or night time sleep) or 3 (symptoms troublesome and interfering with daily activity or night-time sleep). Subjects also recorded sense of smell: 0 (normal), 1 (slightly impaired), 2 (moderately impaired) or 3 (absent).

3. Symptoms of rhinosinusitis. Each subject was asked to indicate the answer to the question "How troublesome are your symptoms of rhinosinusitis?" on a $10 \mathrm{~cm}$ visual analogue scale (VAS) (Not troublesome - Most troublesome imaginable).

4. Nasal endoscopy was performed without the use of decongestants and local anaesthetics using an endoscope with a diameter $\leq 2.7 \mathrm{~mm}$. The signs evaluated included oedema, discharge, crusting, scarring/adhesions and polyps, with each sign rated on a 0-2 scale ${ }^{(10)}$.

5. Peak Nasal Inspiratory Flow (PNIF) was measured using an In-Check portable nasal inspiratory flow meter (Clement Clarke International Ltd, Harlow, Essex, UK). At each assessment the subject inhaled maximally through the nose three times and the highest value was recorded.

6. Acoustic rhinometry was performed (GM Instruments Ltd, Scotland, UK) with the subject in the seated position and stabilization of the head, but without instrument fixation. The minimum cross-sectional area corresponding to the nasal valve (MCA) and the volumes between 2 and $5 \mathrm{~cm}$ (VOL 2-5), 0 to $5 \mathrm{~cm}$ (VOL 0-5), 0 to $7 \mathrm{~cm}$ (VOL 0-7) and 2 to $7 \mathrm{~cm}$ into the nose (VOL 2-7) were recorded.

7. MRI scans of the paranasal sinuses were performed shortly before and after the 12 weeks of treatment. The scan area was from the glabella to the posterior part of the sphenoid sinuses with the subject in the supine position. Axial and coronal SET2 series of scans were performed with the following specification: $3 \mathrm{~mm}, 512$ matrix, FOV 18. The duration of the scan was approximately $15 \mathrm{~min}$. Two experienced experts blinded to treatment status independently scored the MRI's according to defined criteria recently proposed by Hissaria et al. for assessment of MRI's in CRS patients ${ }^{(11)}$.
8. The use of rescue medication (loratidine tablets $10 \mathrm{mg}$ and/or oxymetazoline drops or spray) was recorded in the diary each day throughout the treatment period. A tablet count was used to verify the usage of loratidine tablets.

For assessments 4-6, the order of assessments was acoustic rhinometry, followed by PNIF, then nasal endoscopy.

\section{Safety assessments}

Safety assessments included adverse events, laboratory tests, vital signs and physical examination. Details of all reported adverse events were recorded throughout the study, with severity graded as mild, moderate or severe and a relationship to treatment assigned based on the judgment of the investigator. Blood and urine sampling for laboratory tests, measurement of vital signs and physical examination were performed at screening and follow-up. Blood samples for morning cortisol concentrations were taken between $08.00-10.00 \mathrm{~h}$ at baseline and the end of treatment.

\section{Statistical methods}

All efficacy analyses and summaries are based on the intent-totreat (ITT) population, which included all randomized subjects who received at least one dose of study medication and had baseline and at least one post-baseline measurement.

For efficacy parameters, although data was normally distributed apart from rescue medication, due to the small sample size a non-parametric analysis was used. Results are presented as the median unless otherwise stated. For each variable, data was first tested for an overall treatment effect using the Kruskal-Wallis test. Comparisons of Opt-FP with placebo at each timepoint were then made, where appropriate, using the Mann-Whitney U-test. For sense of smell, due to the large number of equal values/ties the use of the Kruskal-Wallis test was not meaningful. We therefore performed Mann-Whitney U-tests on sense of smell data without a significant overall treatment effect. For MRI, the result for the active treatment group at 12 weeks was additionally compared with the baseline value using the Wilcoxon signed rank test. The level of significance, alpha $(\alpha)$, for this study was 0.05 . All statistical testing was two-sided.

Table 2. Baseline subject demographics and clinical characteristics.

\begin{tabular}{lcc}
\hline Parameter & $\begin{array}{c}\text { Opt-FP } \mathbf{4 0 0} \boldsymbol{\mu g} \text { b.i.d. } \\
(\mathbf{n}=\mathbf{1 0})\end{array}$ & $\begin{array}{c}\text { Placebo b.i.d. } \\
(\mathbf{n}=\mathbf{1 0})\end{array}$ \\
\hline Mean age, years (range ) & $49.2(25-61)$ & $46.7(37-62)$ \\
Male/female (\%) & $60 / 40$ & $80 / 20$ \\
Mean weight, kg (range) & $87.0(64-116)$ & $86.4(68-106)$ \\
Current asthma, n (\%) & $4(40)$ & $3(30)$ \\
Previous sinus surgery, n (\%) & $10(100)$ & $10(100)$ \\
Allergy, n (\%) & $5(50)$ & $5(50)$ \\
ASA intolerance, n (\%) & $3(30)$ & $1(10)$ \\
Median number of sinus & $4(1-10)$ & $3(1-8)$ \\
surgeries (range) & & \\
Previous polypectomy, n (\%) & $2(20)$ & $0(0)$ \\
\hline
\end{tabular}

Opt-FP $=$ OptiNose device containing fluticasone propionate. 
Table 3. Baseline versus post-treatment outcomes: subjective scores.

\begin{tabular}{|c|c|c|c|c|c|c|}
\hline \multirow{3}{*}{ Outcome $^{a}$} & \multicolumn{2}{|c|}{ Opt-FP $(n=9-10)$} & \multicolumn{2}{|c|}{ Placebo $(n=8-10)$} & \multicolumn{2}{|c|}{ p-value ${ }^{b}$} \\
\hline & Baseline & Median & Baseline & Median & Overall & Opt-FP \\
\hline & Median & Change & Median & Change & Treatment & vs \\
\hline & & & & & Effect & Placebo \\
\hline
\end{tabular}

\section{$\underline{\text { RSOM-31 }}$}

Overall Symptoms (31 questions)

\begin{tabular}{|c|c|c|c|c|c|c|}
\hline \\
\hline Baseline & 178.0 & - & 187.0 & - & - & - \\
\hline 12 weeks & - & -62.0 & - & -5.0 & NS & NS \\
\hline \multicolumn{7}{|c|}{ Nasal Symptoms (7 questions) } \\
\hline 4 weeks & - & -11.0 & - & 6.0 & 0.0090 & 0.009 \\
\hline 8 weeks & - & -21.0 & - & 3.0 & & 0.016 \\
\hline 12 weeks & - & -18.0 & - & -1.0 & & NS \\
\hline
\end{tabular}

Nasal Symptom Scores

Combined Symptoms (Morning)

Baseline
4 weeks
8 weeks
12 weeks

Combined Symptoms (Evening)

Baseline

4 weeks

8 weeks

12 weeks

Nasal Discomfort (Morning)

Baseline

12 weeks

Nasal Discomfort (Evening)

Baseline

4 weeks

12 weeks

Sense of Smell (Evening)

Baseline

12 weeks

5.83

- $\quad-1.78$

- $\quad-2.00$

$\begin{array}{ll}- & -2.00\end{array}$

\author{
5.52
}

- $\quad-1.95$

- $\quad-2.00$

$\begin{array}{ll}- & -2.00\end{array}$

2.00

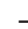

78
.00
.00

\subsection{0}

$-$

$-$

$-$

6.71

$-$

$-$

-

2.29

$-0.67$

2.00

$-$

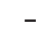

2.17

$+2$

NS

Opt-FP = OptiNose device containing fluticasone propionate; NS = not statistically significant.

${ }^{a}$ Outcome is presented primarily for statistically significant results. Details of non-significant trends for other parameters are presented in the results text.

${ }^{\mathrm{b}}$ p-value: Overall treatment effect determined by Kruskal-Wallis test; comparison of Opt-FP versus placebo determined by Mann-Whitney U test.

\section{RESULTS}

\section{Subjects characteristics}

The study was conducted from September 2007 to September 2008. A total of 20 subjects were randomized to treatment (10 Opt-FP, 10 PBO). The study population was predominantly male and all subjects had a diagnosis of CRS. The two treatment groups were closely similar with respect to demographics and clinical characteristics (Table 2). The intention was to recruit up to 40 subjects, but as in other CRS studies recruitment was slower than anticipated as many eligible subjects were reluctant to risk the possibility of treatment with placebo for three months ${ }^{(11,12)}$. Inclusion was therefore limited to 20 subjects. All 20 randomized subjects were included in the ITT population.
The majority of subjects (9 Opt-FP, 7 PBO) completed the study. Mean percentage compliance was high with $97 \%$ administrations made in the Opt-FP group and 99\% made in the placebo group. Five subjects in the placebo group and 7 in the Opt-FP group chose to continue using nasal saline douche twice daily during the study.

\section{Efficacy}

\section{Subjective Scores}

Subjective scores for CRS (RSOM-31 and nasal symptom scores) are summarized in Table 3.

\section{$\underline{\text { RSOM-31 }}$}

Total RSOM-31 score and all subscale scores showed an improvement trend with Opt-FP treatment. Importantly, the 


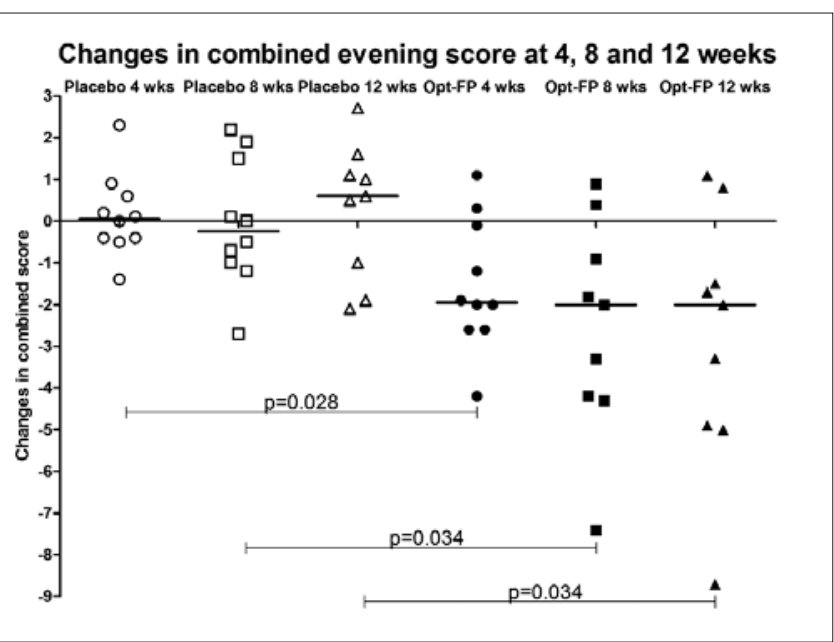

Figure 1. Median change from baseline in evening score for Combined Symptoms after 4, 8 and 12 weeks of treatment. Baseline median was 3.0 for placebo and 4.0 for Opt-FP. Opt-FP $=$ OptiNose device containing fluticasone propionate. Horizontal bars are medians and symbols individual subject data. There was an overall treatment effect by Kruskal-Wallis test $(\mathrm{p}=0.0087)$. Comparison of Opt-FP with placebo was by Mann-Whitney U-test.

nasal RSOM-31 subscale was significantly improved with OptFP treatment compared with placebo. The average reduction in total symptom-impact score was $2(62 / 31)$, and in the nasal subgroup $2.59(18 / 7)$ suggesting a clinically meaningful improvement ${ }^{(9) .}$

\section{$\underline{\text { Subjective symptom scores }}$}

Significant improvements in combined symptom scores were observed for the Opt-FP group compared with placebo at all time points for evening scores (Figure 1) and at 8 weeks $(p=0.030)$ and 12 weeks $(p=0.038)$ for morning scores. This was accompanied by significant improvements in nasal discomfort for the evening score at 4 weeks $(\mathrm{p}=0.044)$ and morning and evening scores at 12 weeks $(\mathrm{p}=0.027)$, and evening score for sense of smell at 12 weeks $(p=0.048)$. Morning and evening scores for nasal blockage and rhinitis showed an improvement trend during Opt-FP treatment, but were not statistically significant compared with placebo.

\section{$\underline{\text { VAS }}$}

Symptoms of rhinosinusitis scored on a VAS showed a nonsignificant improvement trend after 12 weeks of treatment with Opt-FP (Opt-FP -13.0, PBO 2.0, NS).

\section{Objective Scores}

\section{Nasal endoscopy}

Endoscopy score for oedema showed a highly significant improvement over the 12 weeks of Opt-FP treatment (Figure 2). A significant reduction from baseline was observed for nasal discharge in the Opt-FP group, but due to a fairly large effect in the placebo group also, the inter-group difference was not significant. Analysis of changes in crusting, scarring/adhe-

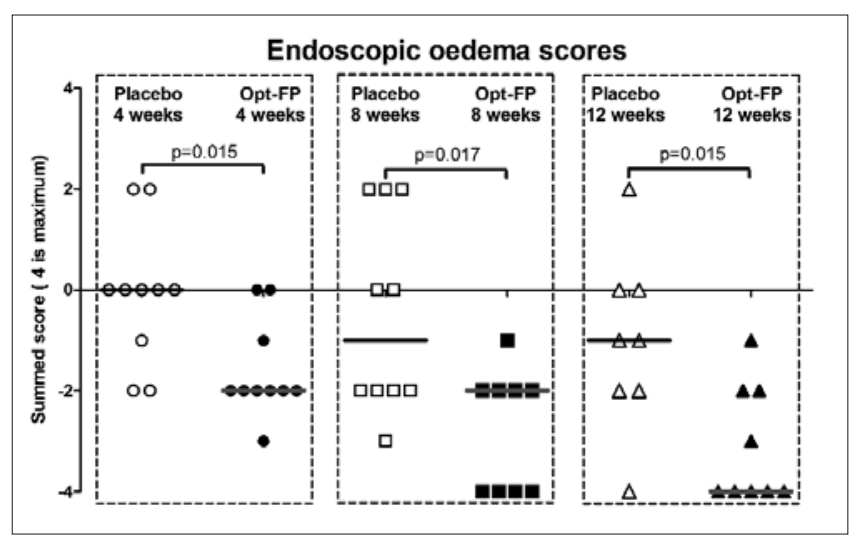

Figure 2. Median change from baseline in Lund and Mackay score for Oedema after 4, 8 and 12 weeks of treatment. Baseline median was 3.0 for placebo and 4.0 for Opt-FP. Opt-FP $=$ OptiNose device containing fluticasone propionate. Horizontal bars are medians and symbols individual subject data. There was an overall treatment effect by KruskalWallis test $(\mathrm{p}=0.0004)$. Comparison of Opt-FP with placebo was by Mann-Whitney U-test.

sions and polyps was not relevant in this non-surgical study excluding polyps ${ }^{(13)}$.

\section{PNIF}

There was a progressive increase in PNIF during Opt-FP treatment with significant differences compared to placebo at 4 weeks ( 35.0 vs $-2.5 \mathrm{~L} / \mathrm{min} \mathrm{p}=0.006)$ and 8 weeks (50.0 vs 10.0 $\mathrm{L} / \mathrm{min}, \mathrm{p}=0.03$ ) and a non-significant increase at 12 weeks (40.0 vs $25.0 \mathrm{~L} / \mathrm{min}, \mathrm{NS}$ ).

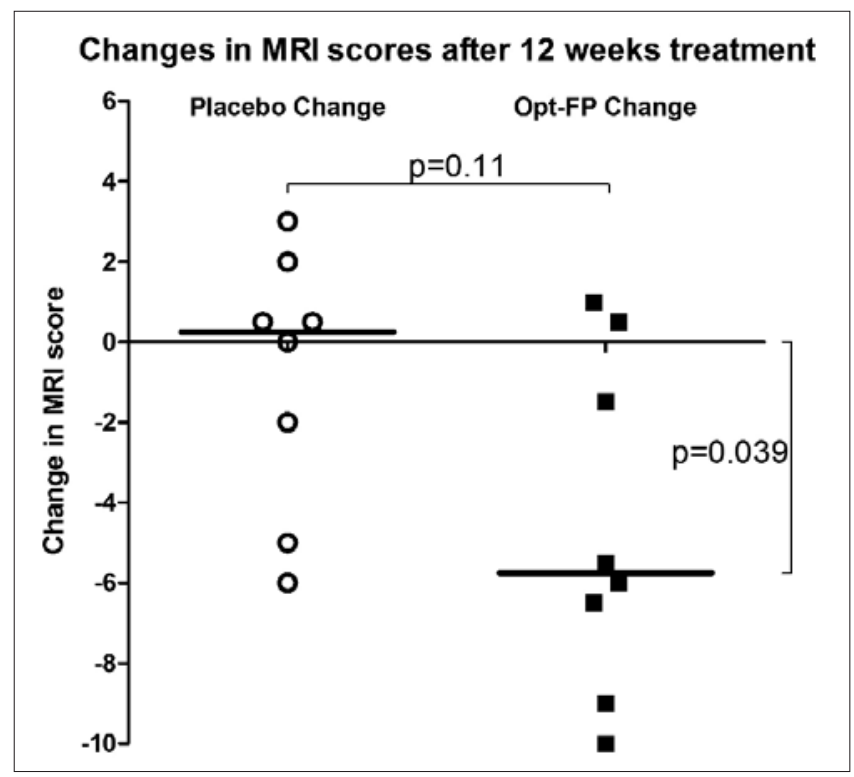

Figure 3. Median change from baseline in MRI score after 12 weeks of treatment. Baseline median was 17.25 for placebo and 19.00 for OptFP. Opt-FP = OptiNose device containing fluticasone propionate. Horizontal bars are medians and symbols individual subject data. Comparison of Opt-FP baseline and 12 week scores was by Wilcoxon signed rank test $(\mathrm{p}=0.039)$. Comparison of Opt-FP and Placebo scores at 12 weeks was by Mann-Whitney U-test $(\mathrm{p}=0.11)$. 


\section{Acoustic rhinometry}

All nasal volumes assessed by acoustic rhinometry (VOL 0-5, VOL 2-5, VOL 0-7 and VOL 2-7) increased considerably with Opt-FP treatment, with a non-significant positive trend. A slight decrease in volumes was observed in the placebo group. The MCA was unchanged.

\section{$\underline{\text { MRI }}$}

MRI results are summarized in Figure 3. The assessment of the MRI's using a new scoring system for MRI suggested by Hissaria et al. ${ }^{(11)}$ showed significant change from baseline in the Opt-FP treated group $(\mathrm{p}=0.039)$. When compared to placebo, a non-significant trend was observed. The correlation between assessments by the two MRI experts was highly significant $(\mathrm{r}=0.91, \mathrm{p}<0.0001)$.

\section{Rescue medication}

Subjects treated with Opt-FP used loratadine on a lower mean percentage of days than subjects treated with placebo over the treatment period $(4.3 \%$ vs $12.1 \%$, NS). Subjects treated with placebo did not use oxymetazoline, whereas there was low use in the Opt-FP group (1.2\% vs $0 \%$, NS).

\section{Safety}

A total of 61 adverse events (Opt-FP $n=25$; PBO $n=36)$ were reported by nine subjects (90\%) in both treatment groups. All were mild or moderate in severity. Adverse events considered to be treatment-related included epistaxis (1 Opt-FP, 2 PBO), nasal polyps (2 $\mathrm{PBO}$ ), rhinorrhoea (1 Opt-FP, $1 \mathrm{PBO}$ ), nasal congestion (1 PBO), sinusitis (1 Opt-FP, 2 PBO) and nasopharyngitis (1 $\mathrm{PBO})$. No serious adverse events were reported during the study. One subject in the Opt-FP group withdrew from treatment due to itchy eyes, headache and redness and swelling of the skin. Two subjects in the placebo group withdrew from treatment due to adverse events, with one subject developing a cough that required concomitant medication and the other suffered an exacerbation of CRS.

The major finding for physical examination was abnormality on ENT examination on screening for all patients in both groups, consistent with their inclusion in the study. At followup, 3 (30.0\%) subjects treated with Opt-FP had an ENT examination assessed as normal, whereas the examination for all placebo-treated subjects was still abnormal. Laboratory test results and vital signs at screening and follow-up showed no clinically relevant changes or treatment effects overall. No adrenal suppression, as assessed by mean morning plasma cortisol concentrations, was apparent for Opt-FP treatment.

\section{DISCUSSION}

The results of this prospective, randomized, placebo controlled study demonstrate that Opt-FP (400 $\mu$ g b.i.d.) is effective in the treatment of CRS, with significant and clinically relevant improvements compared to placebo in both subjective and objective parameters of the disease. It is noteworthy that the patients included in this study had recalcitrant CRS in whom surgery and traditional medical treatment had previously failed. Although a study design with an active comparator would have enabled a direct comparison of a conventional delivery spray with bi-directional delivery, this was not chosen for this small exploratory study, the aim of which was to determine whether the device was at all effective and safe.

There are only very limited data on the treatment of CRS without nasal polyps in the literature. To our knowledge, only two studies with topical steroids in CRS without polyps are available, both from the same group ${ }^{(12,14)}$. In 2001, Parikh et al. ${ }^{(12)}$ reported on a small study from the Royal National Throat, Nose, and Ear Hospital, London, a tertiary referral clinic like the AMC. In this study with fluticasone spray in 22 patients, no significant differences between active and placebo were found after 16 weeks of treatment. The higher FP dose used in our study may be one explanation for the difference. However, the new delivery to target areas beyond the nasal valve, including the middle meatus, is a more likely explanation. A normal nasal spray only reaches the middle meatus in less than half of the patients ${ }^{(5)}$. Inadequate delivery to the target sites in the middle meatus may explain why delivery of a high dose of fluticasone propionate nasal spray ( $800 \mu \mathrm{g}$ b.i.d) for one year following functional endoscopic sinus surgery in patients with CRS with and without polyps did not influence the recurrence rate in any of the subgroups compared to placebo ${ }^{(15)}$. The second study although done by the same group aimed at a totally different patient population. These patients could be considered typical patients initially presenting in primary care with persistent troublesome symptoms of CRS without previous sinus surgery ${ }^{(14)}$. Endoscopy and sinus imaging were not performed in this study. Significant improvements in symptom scores and PNIF were found.

In the two studies using Fluticasone spray and Budesonide spray to treat CRS, $68 \%$ and $57 \%$ had verified allergy compared to $50 \%$ in the present study (Table 2 ) ${ }^{(12,14)}$. In our study $35 \%$ had asthma, which is very similar to the rates reported by Parikh et al. (23\%) and Dijkstra et al. (33\%) (Table 2) ${ }^{(12,14)}$. ASA intolerance was present in $20 \%$ of our patients ( 2 in 10 of the active group), but was not reported or it was an exclusion criteria in the three studies we compare against (Table 2) ${ }^{(12,14,15)}$. The number of previous surgeries was high in our study and similar to Parikh et al. (Table 2) ${ }^{(12,14)}$. Presence of allergy seems to provide a somewhat better clinical outcome for topical steroids, whereas CRS patients with asthma and ASA intolerance and those with repeated surgeries tend to have more severe sinus disease and poorer outcome ${ }^{(2,14)}$. Thus, based on the limited data available, differences in frequency of allergy, asthma, ASA intolerance and previous surgeries cannot explain the positive outcome observed in the present study compared to previous trials.

Numerous attempts have been made to improve the treatment in CRS with other drugs, but many have failed in recent placebo controlled studies leaving patients with significant morbidity and reduced quality of life ${ }^{(16-21)}$. Topical antifungal treat- 
ment with Amphoteracine-B lavage as well as irrigation and nebulization of topical antibiotics were not more effective than saline in recent trials ${ }^{(19,20)}$. Recently, it has been shown that macrolides have anti-inflammatory effects, in addition to the antibiotic effects, which may be beneficial in CRS by inhibiting cytokine production and altering biofilm formation. The first randomized long-term, low-dose macrolide antibiotic study in CRS showed improvement from baseline on some parameters, but no significant improvement over placebo after 12 week treatment ${ }^{(21)}$. Furthermore, the side effects of macrolides and the potential burden on resistance make a local corticosteroid a more attractive treatment.

The reduction at 12 weeks in the average total and nasal subgroup RSOM-31 scores move the median scores from the "bothered a lot" to the "not bothered" disease-impact group and fulfill the criteria for a meaningful clinical effect (average score reduction $>1$ and/or $>30 \%$ score reduction) ${ }^{(9)}$. Excellent, very good or good treatment effects were reported in $80 \%$ of rhinosinusitis patients with a reduction in total RSOM-31 Symptom-Impact score $>30 \%{ }^{(9)}$. This is in agreement with our findings (Figures 1-3).

The highly significant and progressive improvement in the endoscopically observed mucosal oedema is of particular interest. Severe oedema (mean 3.7 / median 4.0 out of 4.0) was significantly reduced to a normal score (mean $0.6 /$ median 0 ) with Opt-FP whereas a smaller non-significant reduction (mean $3.0 \rightarrow 1.8 /$ median $3.0 \rightarrow 1.5$ ) was observed in the placebo group. To our knowledge this has not been described before in the treatment of CRS.

A significant increase in PNIF was observed after 4 weeks (mean $38 \mathrm{~L} / \mathrm{min} /$ median $35 \mathrm{~L} / \mathrm{min}$ ) of treatment and increased with time to a mean of $58 \mathrm{~L} / \mathrm{min} /$ median of 40 $\mathrm{L} / \mathrm{min}$ at 12 weeks from a relatively high baseline PNIF (mean $139 \mathrm{~L} / \mathrm{min} /$ median $132.5 \mathrm{~L} / \mathrm{min}$ ). The mean increase was 49 $\mathrm{L} / \mathrm{min}$ after 20 weeks of treatment with budesonide, but baseline values are not reported ${ }^{(14)}$. Given the large increase in PNIF and reduction in oedema it was expected that nasal volumes measured with acoustic rhinometry would be increased. The volume increases were not statistically significant compared to placebo. Previous sinus surgery often creates artificial openings between the nose and sinuses limiting the usefulness of acoustic rhinometry in CRS patients with previous sinus surgery ${ }^{(22)}$.

Distorted anatomy also complicates the assessment of MRIs, but excellent correlation between examiners suggests a place for MRI diagnoses and follow-up in CRS. The significant improvement in MRI scores in the Opt-FP group compared to baseline and positive trend compared to placebo in this small sample size is noteworthy.

The sinus ostia play a pivotal role in CRS ${ }^{(20)}$. We speculate that the good outcome of the Opt-FP observed both in CRS without polyps and CRS with polyps can be attributed to the superior deposition characteristics to the target site in the middle meatus provided by bi-directional delivery ${ }^{(7,23)}$.
In conclusion, this study presents preliminary evidence that Opt-FP (400 $\mu$ g b.i.d.) is an effective and well tolerated treatment for CRS without nasal polyps or with only cobblestoned mucosa. The results provide hope that the new drug-device combination can address the great unmet need for all the patients suffering from CRS.

\section{ACKNOWLEDGEMENTS}

We thank Dr Nicole Freling (AMC) for performing the MRIs, and Dr Heid Eggesboe and Dr Finn Kolmanskog for further analysis of the MRIs. Ms Tamar Gibson (AMC) provided invaluable assistance with the conduct of the study. Dr Tony Flint, Dr Colin Sheldrake and Dr Graeme Hewson (OptiNose UK Ltd) provided assistance with the study and statistical analyses.

\section{REFERENCES}

1. Fokkens WJ, Lund V, Bachert C. EAACI position paper on rhinosinusitis and nasal polyps executive summary. Allergy 2005; 60: 583-601.

2. Fokkens WJ, Lund VJ, Mullol J et al. European Position Paper on Rhinosinusitis and Nasal Polyps. Rhinology 2007; 45 suppl. 20: 1139.

3. Durr DG, Desrosiers MY, Dassa C. Impact of rhinosinusitis in health care delivery: The Quebec experience. J Otolaryngol 2001; 30: 93-97.

4. Joe SA, Thambi R, Huang J. A systematic review of the use of intranasal steroids in the treatment of chronic rhinosinusitis. Otolaryngol Head Neck Surg 2008; 139: 340-347.

5. Merkus P, Ebbens FA, Muller B, et al. The 'best method' of topical nasal drug delivery: comparison of seven techniques. Rhinology 2006; 44: 102-107.

6. Aggarwal R, Cardozo A, Homer JJ. The assessment of topical nasal drug distribution. Clin Otolaryngol 2004; 29: 201-205.

7. Djupesland PG, Skretting A, Windern M, et al. Breath actuated device improves delivery to target sites beyond the nasal valve. Laryngoscope 2006; 116: 466-472.

8. Aukema AA, Mulder PG, Fokkens WJ. Treatment of nasal polyposis and chronic rhinosinusitis with fluticasone propionate nasal drops reduces need for sinus surgery. J Allergy Clin Immunol 2005; 115: 1017-1023.

9. Piccirillo J, Edwards D, Haiduk A, et al. Psychometric and clinimetric validity of the 31-item Rhinosinusitis Outsome Measure (RSOM-31). Am J Rhinol 1995; 9: 297-306.

10. Lund VJ, Mackay IS. Staging in rhinosinusitis. Rhinology 1993; 31: 183-184.

11. Hissaria P, Smith W, Wormald PJ, et al. Short course of systemic corticosteroids in sinonasal polyposis: A double-blind, randomized, placebo-controlled trial with evaluation of outcome measures. J Allergy Clin Immunol 2006; 118: 128-133.

12. Parikh A, Scadding GK, Darby Y, et al. Topical corticosteroids in chronic rhinosinusitis: a randomized, double-blind, placebo-controlled trial using fluticasone propionate aqueous nasal spray. Rhinology 2001; 39: 75-79.

13. Lund VJ, Kennedy DW. Staging for rhinosinusitis. Otolaryngol Head Neck Surg 1997; 117: S35-S40.

14. Lund VJ, Black JH, Szabó LZ, et al. Efficacy and tolerability of budesonide aqueous nasal spray in chronic rhinosinusitis patients. Rhinology 2004; 42: 57-62.

15. Dijkstra MD, Ebbens FA, Poublon RML, et al. Fluticasone propionate aqueous nasal spray does not influence the recurrence rate of chronic rhinosinusitis and nasal polyps 1 year after functional endoscopic sinus surgery. Clin Exp Allergy 2004; 34: 1395-1400.

16. Fokkens WJ. Editorial: Unmet needs in rhinology. Rhinology 2008; 46: 257-258. 
17. Ebbens FA, Scadding GK, Badia L, et al. Amphotericin B nasal lavages: not a solution for patients with chronic rhinosinusitis. J Allergy Clin Immunol 2006; 118: 1149-1156.

18. Ebbens FA, Georgalas C, Rinia AB, et al. The fungal debate: where do we stand today? Rhinology 2007; 45: 178-189.

19. Liang KL, Su MC, Shiao JY, et al. Amphotericin B irrigation for the treatment of chronic rhinosinusitis without nasal polyps: a randomized, placebo-controlled, double-blind study. Am J Rhinol 2008; 22: 52-58.

20. Videler WJ, van Drunen CM, Reitsma JB, et al. Nebulized bacitracin/colimycin: a treatment option in recalcitrant chronic rhinosinusitis with Staphylococcus aureus? A double-blind, randomized, placebo-controlled, cross-over pilot study. Rhinology 2008; 46: 92 98.

21. Wallwork B, Coman W, Mackay-Sim A, et al. A double-blind, randomized, placebo-controlled trial of macrolide in the treatment of chronic rhinosinusitis. Laryngoscope 2006; 116: 189-193.

22. Djupesland PG, Rotnes JS. Accuracy of acoustic rhinometry. Rhinology 2001; 39: 23-27.
23. Vlckova I, Navrátil P, Kaňa R, et al. Effective treatment of mild-tomoderate nasal polyposis with fluticasone delivered by a novel device. Rhinology 2009; 47: 419-426.

Per G. Djupesland, MD PhD

OptiNose AS

Oslo Innovation Center (Forskningsparken)

Gaustadalleen 21

0349 Oslo

Norway

Tel: $+47-22958015$

Fax: +47-2295 8098

E-mail: pgd@optinose.no 